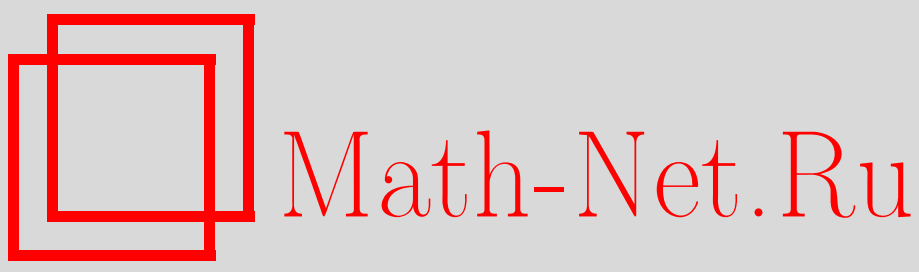

A. I. Budkin, The operator $L_{n}$ on quasivarieties of universal algebras, Sibirsk. Mat. Zh., 2019, Volume 60, Number 4, 724-733

DOI: https://doi.org/10.33048/smzh.2019.60.402

Use of the all-Russian mathematical portal Math-Net.Ru implies that you have read and agreed to these terms of use http://www . mathnet.ru/eng/agreement

Download details:

IP : 3.82 .47 .9

April 26, 2023, 14:13:04

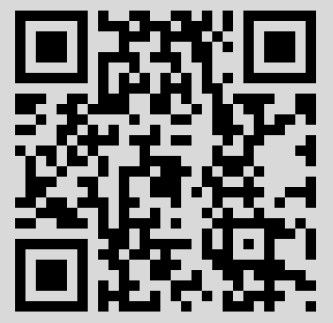


Сибирский математический журнал Июль-август, 2019. Том 60, № 4

УДК 512.57

\title{
ОПЕРАТОР $L_{n}$ НА КВАЗИМНОГООБРАЗИЯХ УНИВЕРСАЛЬНЫХ АЛГЕБР
}

\author{
А. И. Будкин
}

\begin{abstract}
Аннотация. Пусть $n-$ произвольное натуральное число и $\mathscr{M}-$ любой класс универсальных алгебр. Обозначим через $L_{n}(\mathscr{M})$ класс алгебр $G$ таких, что для каждой $n$-порожденной подалгебры $A$ алгебры $G$ смежный класс $a / R(a \in A)$ по наименьшей конгруэнции $R$, содержащей $A \times A$, является алгеброй из $\mathscr{M}$. Исследуются классы $L_{n}(\mathscr{M})$. В частности, доказано, что если $\mathscr{M}$ - квазимногообразие, то $L_{n}(\mathscr{M})$ - квазимногообразие. Аналогичный результат получен для универсально аксиоматизируемых классов алгебр. Также установлено, что если $\mathscr{M}$ - конгруэнцперестановочное многообразие алгебр, то $L_{n}(\mathscr{M})$ является многообразием. Найдено многообразие $P$ полугрупп такое, что класс $L_{1}(P)$ не является многообразием.
\end{abstract}

DOI 10.33048/smzh.2019.60.402

Ключевые слова: квазимногообразие, многообразие, универсальная алгебра, конгруэнц-перестановочное многообразие, класс Леви.

\section{Введение}

Покрытием универсальной алгебры $G$ назовем всякую систему подалгебр этой алгебры, теоретико-множественное объединение подалгебр которой совпадает с $G$. Исследование влияния свойств покрытия на строение самой алгебры одна из важных задач. Весьма подробно эта задача рассматривалась в теории групп. Приведем ряд статей, посвященных решению этой задачи в теории групп, относящихся к классам Леви.

Классы Леви были впервые введены Каппе [1] под влиянием работы Леви [2]. Класс $L(\mathscr{K})$ называется классом Леви, порожденным классом групп $\mathscr{K}$, если он состоит из всех групп $G$, в которых все 1-порожденные (как нормальные) нормальные подгруппы содержатся в $\mathscr{K}$. Оказалось [3], что если $\mathscr{K}$ - многообразие групп, то $L(\mathscr{K})$ также многообразие групп, если $\mathscr{K}$ - квазимногообразие групп, то $L(\mathscr{K})-$ квазимногообразие групп [4]. Дальнейшие свойства классов Леви изучались следующими авторами: Каппе и Морс [5,6], А. И. Будкин [7], А. И. Будкин и Л. В. Таранина [8], В. В. Лодейщикова [9-15], С. А. Шахова [16].

В данной работе сделана попытка обобщения понятия класса Леви, возникшее в теории групп, на случай универсальных алгебр.

Для каждого натурального числа $n$ и всякого класса $\mathscr{M}$ универсальных алгебр мы вводим класс $L_{n}(\mathscr{M})$ следующим образом: $G \in L_{n}(\mathscr{M})$ тогда и только тогда, когда для всякой $n$-порожденной подалгебры $A$ алгебры $G$ смежный класс $a / \theta(a \in A)$ по наименьшей конгруэнции $\theta$, содержащей $A \times A$, является алгеброй из $\mathscr{M}$. Данная работа посвящена исследованию классов $L_{n}(\mathscr{M})$. В частности, доказано, что если $\mathscr{M}$ - квазимногообразие или универсально

(с) 2019 Будкин А. И. 
аксиоматизируемый класс алгебр, то таковым же является класс $L_{n}(\mathscr{M})$. Установлено, что если $\mathscr{M}$ - конгруэнц-перестановочное многообразие алгебр, то $L_{n}(\mathscr{M})$ также является многообразием. Найдено многообразие $\mathscr{P}$ полугрупп такое, что класс $L_{1}(\mathscr{P})$ не является многообразием.

\section{$\S 1$. Предварительные сведения}

Напомним некоторые понятия и определения.

Через $\operatorname{var}(\mathscr{K})$ будем обозначать многообразие, порожденное классом $\mathscr{K}$ алгебр. Всюду в работе через $\mathbb{N}=\{0,1, \ldots\}$ обозначено множество натуральных чисел. Вместо «универсальная алгебра» будем писать просто «алгебра». Сигнатурный символ $f$ и соответствующую ему операцию $f^{A}$ на алгебре $A$ будем обозначать одинаково.

Далее $\langle S\rangle$ - подалгебра, порожденная множеством $S, E$ - одноэлементная алгебра. Запись $H \leq G$ означает, что $H$ - подалгебра алгебры $G$. Вложением алгебры $A$ в алгебру $B$ будем называть любой гомоморфизм $\varphi: A \rightarrow B$, являющийся изоморфизмом $A$ на $\varphi(A)$. Если существует вложение $A$ в $B$, то говорим, что $A$ вложима в $B$.

0 - Трансляиией алгебры $G$ называется тождественное или константное отображение $g: G \rightarrow G$, т. е. функция вида $g(x)=x$ или $g(x)=a$, где $a-$ фиксированный и $x$ - произвольный элементы из $G$. 1-Трансляиией алгебры $G$ называется 0-трансляция или отображение вида

$$
x \rightarrow f\left(a_{1}, \ldots, a_{k-1}, x, a_{k+1}, \ldots, a_{n}\right), \quad x \in G,
$$

где $f$ - символ основной операции алгебры $G$ и $a_{1}, \ldots, a_{n}-$ фиксированные элементы из $G$. Композиция $k 1$-трансляций алгебры $G$ называется $k$-трансляцией этой алгебры. Отображение $g: G \rightarrow G$ называется просто трансляиией алгебры $G$, если $g$ есть $k$-трансляция для некоторого $k ; a / \theta-$ смежный класс по конгруэнции $\theta$, содержащий $a$.

Всюду в работе $\triangle=\{(x, x) \mid x \in G\}$ - тривиальная конгруэнция на $G$.

Пусть $A \leq G$. Через $\theta_{A}$ будем обозначать наименьшую конгруэнцию на $G$, содержащую $A \times A$ (часто вместо $\theta_{A}$ пишут $\theta(A)$ ). Заметим, что при $a \in A$ смежный класс $a / \theta_{A}$ является подалгеброй алгебры $G$.

Отметим, что в случае групп (колец) $a / \theta_{A}(a \in A)$ - это наименьшая нормальная подгруппа группы $G$, содержащая $A$ (наименьший идеал, содержащий $A)$.

Имеет место следующее утверждение [17, следствие 7.10].

Лемма 1. Если $A \leq G$, то $(a, b) \in \theta_{A}$ тогда и только тогда, когда существуют такие пары $\left(x_{i}, y_{i}\right) \in A \times A$ и такие трансляции $g_{i}$ алгебры $G(i=0, \ldots, n)$, что

$$
a=g_{0}\left(x_{0}\right), \quad g_{i}\left(x_{i}\right)=g_{i-1}\left(y_{i-1}\right)(i=1, \ldots, n), \quad g_{n}\left(y_{n}\right)=b .
$$

Пусть $\alpha$ и $\beta-$ бинарные отношения на множестве $G$. Произведение $\alpha \beta$ есть отношение, определяемое следующим образом:

$$
(a, b) \in \alpha \beta \Leftrightarrow(\exists c \in G)(a, c) \in \alpha \text { и }(c, b) \in \beta .
$$

Класс алгебр $\mathscr{K}$ называется конгруэнц-перестановочным, если во всякой алгебре $G \in \mathscr{K}$ любые две конгруэнции $\theta$ и $\rho$ перестановочны (т. е. $\theta \rho=\rho \theta$ ). Класс алгебр $\mathscr{K}$ имеет $n$-перестановочные конгруэнции, если во всякой алгебре 
$G \in \mathscr{K}$ для любых конгруэнций $\theta$ и $\rho$ имеет место $\theta \vee \rho=\theta \rho \theta \rho \ldots$ ( $n$ сомножителей). Класс алгебр $\mathscr{K}$ называется конгруэни-дистрибутивным, если для всякой алгебры $G \in \mathscr{K}$ ее решетка конгруэнций дистрибутивна. Класс алгебр $\mathscr{K}$ называется арифметическим, если он конгруэнц-перестановочный и конгруэнц-дистрибутивный.

Пусть $\left\{A_{i} \mid i \in I\right\}-$ непустое семейство алгебр $A_{i}$. Рассмотрим декартово произведение $A=\left\{a: I \rightarrow \cup A_{i} \mid a(i) \in A_{i}\right\}$ их носителей. Определив операции на $A$ так:

$$
f\left(a_{1}, \ldots, a_{n}\right)(i)=f\left(a_{1}(i), \ldots, a_{n}(i)\right) \quad \text { для всех } i \in I,
$$

получаем прямое произведение этих алгебр, которое обозначается через $\prod_{i \in I} A_{i}$. Пусть $\mathscr{D}$ - произвольный фильтр над $I, \vartheta$ - конгруэнция на $A=\prod_{i \in I} A_{i}$, определенная таким образом:

$$
a \vartheta b \Leftrightarrow\{i \in I \mid a(i)=b(i)\} \in \mathscr{D} .
$$

Тогда фактор-алгебра $A / \vartheta$ называется фильтрованным произведением алгебр $A_{i}$ nо фильтру $\mathscr{D}$ и обозначается через $\prod_{i \in I} A_{i} / \mathscr{D}$. Смежный класс $a / \vartheta$ принято обозначать через $a \mathscr{D}$.

Класс алгебр $\mathscr{K}$ называется наследственным, если с каждой своей алгеброй содержит все ее подалгебры; гомоморфно замкнутым, если с каждой своей алгеброй содержит все ее гомоморфные образы; мультипликативно замкнутым, если содержит всевозможные прямые произведения алгебр из $\mathscr{K}$; замкнутым относительно фильтрованных произведений, если содержит всевозможные фильтрованные произведения алгебр из $\mathscr{K}$.

Говорят, что аксиоматический ранг квазимногообразия $\mathscr{K}$ в данном классе $\mathscr{N}(\mathscr{K} \subseteq \mathscr{N})$ равен $n$, если $\mathscr{K}$ можно задать в $\mathscr{N}$ системой квазитождеств от $n$ переменных и нельзя задать никаким множеством квазитождеств от меньшего числа переменных. Если такого числа $n$ не существует, то $\mathscr{K}$ имеет бесконечный аксиоматический ранг в $\mathscr{N}$. В качестве $\mathscr{N}$ будем рассматривать класс всех алгебр данной сигнатуры.

Будем пользоваться следующим известным утверждением, вытекающим из определения фильтрованного произведения.

Лемма 2. Пусть $t_{1}\left(x_{1}, \ldots, x_{n}\right), t_{2}\left(x_{1}, \ldots, x_{n}\right)$ - произвольные термы рассматриваемой сигнатуры, $\mathscr{D}-$ фильтр над $I, A=\prod_{i \in I} A_{i} / \mathscr{D}, a_{1} \mathscr{D}, \ldots, a_{n} \mathscr{D} \in A$. Справедливо следующее соотношение:

$$
\begin{aligned}
t_{1}\left(a_{1} \mathscr{D}, \ldots, a_{n} \mathscr{D}\right)= & t_{2}\left(a_{1} \mathscr{D}, \ldots, a_{n} \mathscr{D}\right) \\
& \Leftrightarrow\left\{i \in I \mid t_{1}\left(a_{1}(i), \ldots, a_{n}(i)\right)=t_{2}\left(a_{1}(i), \ldots, a_{n}(i)\right)\right\} \in \mathscr{D} .
\end{aligned}
$$

Пусть $\mathscr{M}$ - любой класс алгебр, $n(n \geq 1)-$ произвольное натуральное число. Тогда через $L_{n}(\mathscr{M})$ обозначим класс всех алгебр $G$, обладающих следующим свойством: для любой $n$-порожденной подалгебры $A$ алгебры $G$ подалгебра $a / \theta_{A}(a \in A)$ принадлежит классу $\mathscr{M}$.

Отметим, что в случае групп класс $L_{1}(\mathscr{M})$ совпадает с классом Леви, порожденным классом $\mathscr{M}[1,4]$. 


\section{$\S 2$. Общие свойства оператора $L_{n}$}

Лемма 3. Если $\mathscr{M}$ - наследственный класс алгебр, то $L_{n}(\mathscr{M})$ также является наследственным.

ДокАЗАТЕЛЬСтво. Покажем, что класс $L_{n}(\mathscr{M})$ вместе с каждой алгеброй $G$ содержит все ее подалгебры. Пусть $H \leq G$ и $A-n$-порожденная подалгебра, $A \leq H, \theta_{A}^{\prime}$ и $\theta_{A}$ - определенные ранее конгруэнции на $H$ и $G$ соответственно. Из определений $\theta_{A}^{\prime}$ и $\theta_{A}$ и леммы 1 следует, что $\theta_{A}^{\prime} \subseteq \theta_{A}$, откуда $a / \theta_{A}^{\prime} \leq a / \theta_{A}$ при $a \in A$. Поскольку $a / \theta_{A} \in \mathscr{M}$ и $\mathscr{M}-$ наследственный класс, то $a / \theta_{A}^{\prime} \in \mathscr{M}$. Отсюда $H \in L_{n}(\mathscr{M})$. Лемма доказана.

Теорема 1. Если $\mathscr{M}-$ квазимногообразие алгебр, то $L_{n}(\mathscr{M})$ также является квазимногообразием.

ДокАЗАтЕльство. Ясно, что единичная алгебра $E$ содержится в классе $L_{n}(\mathscr{M})$. По лемме $3 L_{n}(\mathscr{M})-$ наследственный класс. Осталось ввиду теоремы Мальцева $\left[18, \S 11\right.$, следствие 3] показать замкнутость класса $L_{n}(\mathscr{M})$ относительно фильтрованных произведений.

Пусть $G_{k} \in L_{n}(\mathscr{M})(k \in K), \mathscr{D}$ - фильтр над $K, G=\prod_{k \in K} G_{k} / \mathscr{D}$ - фильтрованное произведение алгебр $G_{k}$ по фильтру $\mathscr{D}, A=\left\langle f_{1} \mathscr{D}, \ldots, f_{n} \mathscr{D}\right\rangle \leq G, a \mathscr{D}-$ произвольный элемент из $A$. Необходимо показать, что $a \mathscr{D} / \theta_{A} \in \mathscr{M}$. Предположим, что $A_{k}=\left\langle f_{1}(k), \ldots, f_{n}(k)\right\rangle$ - подалгебра алгебры $G_{k}$, порожденная элементами $f_{1}(k), \ldots, f_{n}(k)$, а $f_{1}(k) / \theta_{A_{k}}$ - соответствующие смежные классы (заметим, что вместо $f_{1}(k)$ можно взять любой элемент из $A_{k}$ ). Рассмотрим алгебру $B=\prod_{k \in K}\left(f_{1}(k) / \theta_{A_{k}}\right) / \mathscr{D}$. Зададим отображение $\varphi: a \mathscr{D} / \theta_{A} \rightarrow B$ следующим образом.

Берем любой элемент $b \mathscr{D} \in a \mathscr{D} / \theta_{A}$. Тогда $(a \mathscr{D}, b \mathscr{D}) \in \theta_{A}$. По лемме 1 существуют такие пары $\left(x_{i} \mathscr{D}, y_{i} \mathscr{D}\right) \in A \times A$ и такие трансляции $g_{i}$ алгебры $G$ $(i=0, \ldots, s)$, что

$$
a \mathscr{D}=g_{0}\left(x_{0} \mathscr{D}\right), \quad g_{i}\left(x_{i} \mathscr{D}\right)=g_{i-1}\left(y_{i-1} \mathscr{D}\right)(i=1, \ldots, s), \quad g_{s}\left(y_{s} \mathscr{D}\right)=b \mathscr{D} .
$$

Обозначим через $J$ множество всех $j \in K$ таких, что в алгебре $G_{j}$ имеем

$$
\begin{gathered}
a(j), x_{i}(j), y_{i}(j) \in A_{j} \quad(i=0, \ldots, s), \\
a(j)=g_{0}\left(x_{0}(j)\right), \quad g_{i}\left(x_{i}(j)\right)=g_{i-1}\left(y_{i-1}(j)\right)(i=1, \ldots, s), \quad g_{s}\left(y_{s}(j)\right)=b(j),
\end{gathered}
$$

где $g_{i}-$ соответствующие трансляции алгебры $G_{j}(i=0, \ldots, s)$. Из замкнутости фильтра относительно пересечений конечного числа своих элементов следует, что $J \in \mathscr{D}$. Из леммы 1 вытекает, что при каждом $j \in J(a(j), b(j)) \in \theta_{A_{j}}$, т. е. $b(j) \in a(j) / \theta_{A_{j}}$. Поскольку $a(j) / \theta_{A_{j}}=f_{1}(j) / \theta_{A_{j}}$, можем определить следующий элемент $t_{b} \in \prod_{k \in K}\left(f_{1}(k) / \theta_{A_{k}}\right)$ :

$t_{b}(j)=b(j)$ при $j \in J ; \quad t_{b}(j)$ - произвольный элемент из $f_{1}(j) / \theta_{A_{j}}$ при $j \notin J$.

Полагаем по определению

$$
\varphi(b \mathscr{D})=t_{b} \mathscr{D} .
$$

Отметим, что если при построении $\varphi(b \mathscr{D})$ пользоваться другим набором элементов $a \mathscr{D}, x_{i} \mathscr{D}, y_{i} \mathscr{D} \in A(i=0, \ldots, m)$ и другим набором трансляций $g_{i}$ алгебры $G(i=0, \ldots, m)$, то по аналогии с $J$ возникнет некоторое множество $J^{\prime} \in \mathscr{D}$, по которому определяется $t_{b}$. Поскольку $J \cap J^{\prime} \in \mathscr{D}$, образ элемента $b \mathscr{D}$ задается однозначно. 
Покажем, что $\varphi-$ гомоморфизм. Пусть $f-m$-арная операция, $b_{1} \mathscr{D}, \ldots$, $b_{m} \mathscr{D} \in a \mathscr{D} / \theta_{A}$. Так как $\mathscr{D}-$ фильтр, из определения $t_{b}$ и леммы 2 следует, что существует можество $J_{1} \in \mathscr{D}$ такое, что $t_{b_{i}}(j)=b_{i}(j)$ для всех $j \in J_{1}$ и каждого $i=1, \ldots, m$. Тогда

$$
f\left(b_{1}, \ldots, b_{m}\right)(j)=f\left(b_{1}(j), \ldots, b_{m}(j)\right)=f\left(t_{b_{1}}(j), \ldots, t_{b_{m}}(j)\right)=f\left(t_{b_{1}}, \ldots, t_{b_{m}}\right)(j)
$$

для каждого $j \in J_{1}$. Возьмем $J_{2} \in \mathscr{D}$ такое, что

$$
t_{f\left(b_{1}, \ldots, b_{m}\right)}(j)=f\left(b_{1}, \ldots, b_{m}\right)(j)
$$

для всех $j \in J_{2}$. Тогда при каждом $j \in J_{1} \cap J_{2} \in \mathscr{D}$ имеем

$$
t_{f\left(b_{1}, \ldots, b_{m}\right)}(j)=f\left(t_{b_{1}}, \ldots, t_{b_{m}}\right)(j) .
$$

Это означает, что $t_{f\left(b_{1}, \ldots, b_{m}\right)} \mathscr{D}=f\left(t_{b_{1}}, \ldots, t_{b_{m}}\right) \mathscr{D}$, откуда

$$
\begin{aligned}
\varphi\left(f\left(b_{1} \mathscr{D}, \ldots, b_{m} \mathscr{D}\right)\right)=\varphi\left(f\left(b_{1}, \ldots, b_{m}\right) \mathscr{D}\right)=t_{f\left(b_{1}, \ldots, b_{m}\right)} \mathscr{D} \\
=f\left(t_{b_{1}}, \ldots, t_{b_{m}}\right) \mathscr{D}=f\left(t_{b_{1}} \mathscr{D}, \ldots, t_{b_{m}} \mathscr{D}\right)=f\left(\varphi\left(b_{1} \mathscr{D}\right), \ldots, \varphi\left(b_{m} \mathscr{D}\right)\right) .
\end{aligned}
$$

Таким образом, $\varphi$ - гомоморфизм.

Покажем, что $\varphi-$ вложение. Пусть $\varphi\left(b_{1} \mathscr{D}\right)=\varphi\left(b_{2} \mathscr{D}\right)$. Поскольку $\mathscr{D}-$ фильтр, найдется $J \in \mathscr{D}$ такое, что

$$
b_{1}(j)=t_{b_{1}}(j)=t_{b_{2}}(j)=b_{2}(j) \quad \text { для всех } j \in J .
$$

Отсюда $b_{1} \mathscr{D}=b_{2} \mathscr{D}$, следовательно, $\varphi-$ вложение.

Так как $A_{k}=\left\langle f_{1}(k), \ldots, f_{n}(k)\right\rangle-n$-порожденная подалгебра алгебры $G_{k} \in$ $\mathscr{M}$, то $f_{1}(k) / \theta_{A_{k}} \in \mathscr{M}$. Значит, $B=\prod_{k \in K}\left(f_{1}(k) / \theta_{A_{k}}\right) \mathscr{D} \in \mathscr{M}$, откуда $a \mathscr{D} / \theta_{A} \in \mathscr{M}$, поскольку $\varphi-$ вложение. Теорема доказана.

Несложная модификация доказательства теоремы 1 приводит нас к следующим результатам.

Следствие 1. Если $\mathscr{M}$ - наследственный и мультипликативно замкнутый класс алгебр, то $L_{n}(\mathscr{M})$ наследственный и мультипликативно замкнутый.

ДокАЗАТЕЛЬСтво. Наследственность класса $L_{n}(\mathscr{M})$ вытекает из леммы 3. Далее следует повторить доказательство теоремы 1 , взяв в качестве $\mathscr{D}$ одноэлементный фильтр $\mathscr{D}=\{K\}$. Известно, что в этом случае фильтрованное произведение над $\mathscr{D}$ совпадает с прямым произведением над $K$. Следствие доказано.

Следствие 2. Если $\mathscr{M}$ - наследственный и ультразамкнутый класс алгебр, то $L_{n}(\mathscr{M})$ наследственный и ультразамкнутый.

ДокАЗАТЕЛЬСтво. Следует повторить доказательство теоремы 1 , взяв в качестве $\mathscr{D}$ ультрафильтр. Следствие доказано.

Известно $[18, \S 8.3$, следствие 5], что класс алгебр универсально аксиоматизируем тогда и только тогда, когда он ультразамкнутый и наследственный. Значит, следствие 2 можно переформулировать следующим образом.

Теорема 2. Если $\mathscr{M}$ - универсально аксиоматизируемый класс алгебр, то $L_{n}(\mathscr{M})$ также является универсально аксиоматизируемым классом.

В случае групп известно [14, лемма 1], что если $\mathscr{M}$ - локально конечное квазимногообразие групп, то класс $L_{1}(\mathscr{M})$ также локально конечен. Аналог этого результата оказался несправедливым в случае универсальных алгебр. 
Теорема 3. Существует локально конечное квазимногообразие $\mathscr{P}_{0}$ алгебр такое, что $L_{1}\left(\mathscr{P}_{0}\right)$ не является локально конечным.

ДокАЗАТЕЛЬСтво. Возьмем в качестве $\mathscr{P}_{0}$ многообразие полурешеток. Ясно, что $\mathscr{P}_{0}$ - локально конечное многообразие.

Рассмотрим следующий 2-порожденный группоид $G$. Его элементы таковы: $0, x_{1}, a_{1}, a_{2}, a_{3}, \ldots$, где $a_{1}=x_{2}, a_{2}=x_{2} x_{1}, a_{3}=a_{2} x_{1}, \ldots, a_{i+1}=a_{i} x_{1}, \ldots$ Операции заданы следующим образом:

$$
\begin{gathered}
x_{1} \cdot x_{1}=x_{1}, \quad 0 \cdot x=x \cdot 0=0 \quad \text { для всех } x \in G, \\
a_{i} \cdot x_{1}=a_{i+1}, \quad a_{i} \cdot a_{i}=a_{i} \quad(i=1,2, \ldots), \\
u \cdot v=0 \quad \text { для всех остальных пар }(u, v) .
\end{gathered}
$$

Каждая 1-порожденная подалгебра $A$ алгебры $G$ является одноэлементной полурешеткой, поэтому $\theta_{A}=\Delta$, откуда $G \in L_{1}\left(\mathscr{P}_{0}\right)$. Поскольку $G-2$-порожденная бесконечная алгебра, $L_{1}\left(\mathscr{P}_{0}\right)$ не является локально конечным классом. Теорема доказана.

Было бы интересно найти условия на классы алгебр, при выполнении которых из локальной конечности квазимногообразия $\mathscr{M}$ следует локальная конечность класса $L_{n}(\mathscr{M})$.

Из определения $L_{n}(\mathscr{M})$ вытекает, что если $\mathscr{M}$ - наследственный класс, то

$$
L_{1}(\mathscr{M}) \supseteq L_{2}(\mathscr{M}) \supseteq L_{3}(\mathscr{M}) \cdots \supseteq \mathscr{M} .
$$

Если $\mathscr{M}$ - квазимногообразие аксиоматического ранга $n$, то алгебра $G$ принадлежит $\mathscr{M}$ тогда и только тогда, когда все ее $n$-порожденные подалгебры содержатся в $\mathscr{M}$. Отсюда $L_{n}(\mathscr{M})=\mathscr{M}$ в этом случае. Возникает вопрос: если $\mathscr{M}$ - квазимногообразие алгебр и $L_{n}(\mathscr{M})=\mathscr{M}$ при подходящем $n$, верно ли, что $\mathscr{M}$ имеет конечный аксиоматический ранг? Это верно, например, для всякого квазимногообразия абелевых групп.

Поскольку все $n$-порожденные алгебры из квазимногообразия $\mathscr{M}$ содержатся в $L_{n}(\mathscr{M})$ и всякое квазимногообразие порождается своими конечно порожденными алгебрами, получаем

Предложение 1. Если $\mathscr{M}$ - квазимногообразие алгебр, то

$$
\bigcap_{n<\omega} L_{n}(\mathscr{M})=\mathscr{M} .
$$

Отсюда вытекает

Следствие 3. Если $\mathscr{M}, \mathscr{N}-$ квазимногообразия алгебр и $\mathscr{M} \neq \mathscr{N}$, то найдется натуральное число $n$ такое, что $L_{n}(\mathscr{M}) \neq L_{n}(\mathscr{N})$.

ДоКАЗАТЕЛЬСтво. Если предположить, что $L_{n}(\mathscr{M})=L_{n}(\mathscr{N})$ для каждого $n$, то

$$
\mathscr{M}=\bigcap_{n<\omega} L_{n}(\mathscr{M})=\bigcap_{n<\omega} L_{n}(\mathscr{N})=\mathscr{N}
$$

ввиду предложения 1. Полученное противоречие доказывает следствие. 


\section{§ 3. Многообразия алгебр и оператор $L_{n}$}

Ясно, что если квазитождество от $n$-переменных истинно в каждой алгебре из квазимногообразия $\mathscr{M}$, то оно истинно в $L_{n}(\mathscr{M})$. Ряд свойств многообразий описывается на языке тождеств (например, при помощи условий Мальцева [17]). Применяя эти описания, получаем следующее утверждение.

Теорема 4. 1. Если $\mathscr{M}$ - конгруэнц-перестановочное или арифметическое многообразие алгебр, то таковым же является квазимногообразие $L_{2}(\mathscr{M})$.

2. Если многообразие алгебр $\mathscr{M}$ имеет $n$-перестановочные конгруэнции или является конгруэнц-дистрибутивным или конгруэнц-модулярным, то таковым же классом является квазимногообразие $L_{3}(\mathscr{M})$.

ДоказатЕЛЬСтво. Известно [17, теоремы 13.1, 13.2, 14.1, 46.1], что многообразие $\mathscr{M}$ обладает свойством из формулировки теоремы тогда и только тогда, когда в нем выполнен определенный набор тождеств (от двух переменных в случае 1 , от трех переменных в случае 2). Значит, эти тождества истинны в свободных алгебрах из соответствующих квазимногообразий $L_{k}(\mathscr{M})(k \geq 2$ в случае 1 , $k \geq 3$ в случае 2$)$. Но свободные алгебры в классах $L_{k}(\mathscr{M})$ и $\operatorname{var}\left(L_{k}(\mathscr{M})\right)$ одни и те же. Поэтому рассматриваемые тождества истинны в соответствующих многообразиях $\operatorname{var}\left(L_{k}\left(\mathscr{M}_{i}\right)\right)$, следовательно, эти многообразия (и их подквазимногообразия $\left.L_{k}\left(\mathscr{M}_{i}\right)\right)$ обладают свойствами из формулировки теоремы. Теорема доказана.

Теорема 5. Существует конгруэнц-перестановочное многообразие алгебр $\mathscr{M}$ такое, что квазимногообразие $L_{1}(\mathscr{M})$ не конгруэнц-перестановочно.

ДокАЗАТЕЛЬСтво. Возьмем в качестве $\mathscr{M}$ многообразие алгебр, заданное тождествами

$$
m(x, x, y)=y, \quad m(x, y, y)=x,
$$

где $m-3$-арная операция. По теореме Мальцева [17, теорема 13.1] многообразие $\mathscr{M}$ конгруэнц-перестановочное.

Пусть $\mathscr{N}$ - многообразие алгебр, заданное тождеством $m(x, x, x)=x$. Если $G \in L_{1}(\mathscr{M}), a \in G$, то $a / \theta_{\langle a\rangle} \in \mathscr{M}$. Поскольку в $\mathscr{M}$ истинно тождество $m(x, x, x)=x$, это тождество истинно в $a / \theta_{\langle a\rangle}$, откуда $m(a, a, a)=a$. Значит, $L_{1}(\mathscr{M}) \subseteq \mathscr{N}$.

Пусть $G \in \mathscr{N}$. Тогда всякая ее 1-порожденная подалгебра будет одноэлементной. Отсюда наименьшей конгруэнцией, содержащей $\langle a\rangle \times\langle a\rangle$, будет $\Delta$, откуда $a / \theta_{\langle a\rangle}=\langle a\rangle \in \mathscr{M}$. Следовательно, $G \in L_{1}(\mathscr{M})$, т. е. $\mathscr{N} \subseteq L_{1}(\mathscr{M})$. Итак, $\mathscr{N}=L_{1}(\mathscr{M})$.

Пусть $\langle P, \cdot\rangle$ - свободная полурешетка ранга $3, a, b, c$ - ее свободные порождающие, $\theta(x, y)$ - конгруэнция на ней, порожденная парой $(x, y)$. Несложно проверяется, что $(a, c) \in \theta(a, b) \theta(b, c)$ и $(a, c) \notin \theta(b, c) \theta(a, b)$, откуда $\theta(a, b) \theta(b, c) \neq$ $\theta(b, c) \theta(a, b)$. Введем на этой полурешетке термальную операцию так: $f(x, y, z)$ $=x y z$. Возникла алгебра $\langle P, f\rangle \in \mathscr{N}$. Поскольку $f$ - термальная операция, всякая конгруэнция на $\langle P, \cdot\rangle$ является конгруэнцией на $\langle P, f\rangle$. Следовательно, конгруэнции на $\langle P, f\rangle$ не перестановочны. Теорема доказана.

Лемма 4. Если $\mathscr{M}$ - конгруэнц-перестановочный и гомоморфно замкнутый класс алгебр, то класс $L_{n}(\mathscr{M})$ гомоморфно замкнут.

ДоказАтЕЛЬСтво. Поскольку $E \in L_{n}(\mathscr{M})$, то $L_{n}(\mathscr{M}) \neq \varnothing$. Пусть $G \in$ $L_{n}(\mathscr{M}), \varphi: G \rightarrow B$ - гомоморфизм алгебры $G$ на $B, \theta=\operatorname{ker} \varphi=\{(x, y) \mid \varphi(x)=$ $\varphi(y)\}$ - ядро гомоморфизма $\varphi$. Образ элемента $x \in G$ при гомоморфизме $\varphi$ 
удобно обозначать через $\bar{x}$. Пусть $\bar{A}=\left\langle\bar{a}_{1}, \ldots, \bar{a}_{n}\right\rangle-n$-порожденная подалгебра алгебры $B, A=\left\langle a_{1}, \ldots, a_{n}\right\rangle \leq G$.

По лемме $1\left(a_{1}, d\right) \in \theta_{A}$ тогда и только тогда, когда существуют такие пары $\left(x_{i}, y_{i}\right) \in A \times A$ и такие трансляции $h_{i}$ алгебры $G(i=0, \ldots, s)$, что

$$
a_{1}=h_{0}\left(x_{0}\right), \quad h_{i}\left(x_{i}\right)=h_{i-1}\left(y_{i-1}\right)(i=1, \ldots, s), \quad h_{s}\left(y_{n}\right)=d .
$$

Рассмотрев образы всех только что выписанных элементов, видим, что если $d \in a_{1} / \theta_{A}$, то $\varphi(d) \in \bar{a}_{1} / \theta_{\bar{A}}$. Таким образом, $\varphi\left(a_{1} / \theta_{A}\right) \subseteq \bar{a}_{1} / \theta_{\bar{A}}$. Докажем обратное включение.

Возьмем произвольный элемент $\bar{b} \in \bar{a}_{1} / \theta_{\bar{A}}$. По лемме 1 существуют такие пары $\left(\bar{x}_{i}, \bar{y}_{i}\right) \in \bar{A} \times \bar{A}$ и такие трансляции $\bar{g}_{i}$ алгебры $B(i=0, \ldots, s)$, что

$$
\begin{gathered}
\bar{a}_{1}=\bar{g}_{0}\left(\bar{x}_{0}\right), \bar{g}_{1}\left(\bar{x}_{1}\right)=\bar{g}_{0}\left(\bar{y}_{0}\right), \ldots, \bar{g}_{i}\left(\bar{x}_{i}\right)=\bar{g}_{i-1}\left(\bar{y}_{i-1}\right), \ldots, \bar{g}_{s}\left(\bar{x}_{s}\right)=\bar{g}_{s-1}\left(\bar{y}_{s-1}\right), \\
\bar{g}_{s}\left(\bar{y}_{s}\right)=\bar{b}
\end{gathered}
$$

Пусть $g_{i}-$ соответствующая трансляция алгебры $G$ такая, что $\varphi\left(g_{i}(x)\right)=$ $\bar{g}_{i}(\bar{x})$. Считаем, что все $x_{i}, y_{i}$ взяты из $A$. Отсюда $\left(g_{i}\left(x_{i}\right), g_{i}\left(y_{i}\right)\right) \in \theta_{A}$.

Применив выписанное выше к алгебре $G$, имеем

$$
\begin{gathered}
\left(a_{1}, g_{0}\left(x_{0}\right)\right) \in \theta, \quad\left(g_{0}\left(y_{0}\right), g_{1}\left(x_{1}\right)\right) \in \theta, \quad\left(g_{1}\left(y_{1}\right), g_{2}\left(x_{2}\right)\right) \in \theta, \ldots, \\
\left(g_{s-1}\left(y_{s-1}\right), g_{s}\left(x_{s}\right)\right) \in \theta, \quad\left(g_{s}\left(y_{s}\right), b\right) \in \theta .
\end{gathered}
$$

Учитывая, что $\left(g_{i}\left(x_{i}\right), g_{i}\left(y_{i}\right)\right) \in \theta_{A}$, получаем

$$
\left(a_{1}, b\right) \in \theta \theta_{A} \theta \theta_{A} \ldots \theta_{A} \theta .
$$

Поскольку конгруэнции на алгебре $G$ перестановочны, имеем $\left(a_{1}, b\right) \in \theta_{A} \theta$. В силу определения произведения конгруэнций найдется элемент $c \in G$ такой, что $\left(a_{1}, c\right) \in \theta_{A},(c, b) \in \theta$. Следовательно, $\varphi(c)=\bar{b}$ и $c \in a_{1} / \theta_{A}$.

Итак, всякий элемент из $\bar{a}_{1} \theta_{\bar{A}}$ имеет прообраз, содержащийся в $a_{1} / \theta_{A}$. Значит, $\bar{a}_{1} / \theta_{\bar{A}}-$ образ некоторой подалгебры алгебры $a_{1} / \theta_{A}$ при $\varphi$. Из $\varphi\left(a_{1} / \theta_{A}\right) \subseteq$ $\bar{a}_{1} / \theta_{\bar{A}}$, получаем, что $\varphi\left(a_{1} / \theta_{A}\right)=\bar{a}_{1} / \theta_{\bar{A}}$. Поскольку $A-n$-порожденная алгебpa, $a_{1} / \theta_{A} \in \mathscr{M}$. Но $\mathscr{M}-$ гомоморфно замкнутый класс, значит, $\bar{a}_{1} / \theta_{\bar{A}} \in \mathscr{M}$. Итак, показали, что $B \in L_{n}(\mathscr{M})$. Лемма доказана.

Теорема 6. Если $\mathscr{M}$ - конгруэнц-перестановочное многообразие алгебр, то $L_{n}(\mathscr{M})$ также является многообразием.

ДокАЗАТЕЛЬСтво. По известной теореме Биркгофа [18] класс алгебр является многообразием тогда и только тогда, когда он наследствен, мультипликативно и гомоморфно замкнут. Из следствия 1 и леммы 4 вытекает, что наследственным, мультипликативно и гомоморфно замкнутым является класс $L_{n}(\mathscr{M})$. Следовательно, $L_{n}(\mathscr{M})$ - многообразие. Теорема доказана.

Отметим, что известными [17] примерами конгруэнц-перестановочных многообразий служат многообразия групп, колец, квазигрупп, луп, булевых алгебр.

Следующий пример показывает, что в формулировке теоремы 6 избавиться от конгруэнц-перестановочности нельзя. 
Теорема 7. Пусть Я - многообразие коммутативных полугрупп. Тогда $L_{1}(\mathscr{P})$ не является многообразием.

ДокАЗАТЕЛЬСтво. Пусть $F=\langle a, b\rangle$ - свободная полугруппа со свободными порождающими $a, b, A=\langle x\rangle$. Покажем сначала, что $x / \theta_{A} \in \mathscr{P}$. Рассмотрим

$$
\begin{gathered}
\theta=\Delta \cup\left\{\left(x^{k_{1}} a^{m_{1}} b^{n_{1}} x^{k_{2}} a^{m_{2}} b^{n_{2}} \ldots x^{k_{s}} a^{m_{s}} b^{n_{s}}, x^{r_{1}} a^{m_{1}} b^{n_{1}} x^{r_{2}} a^{m_{2}} b^{n_{2}} \ldots x^{r_{s}} a^{m_{s}} b^{n_{s}}\right)\right. \\
\left.\mid(\exists i, j)\left(k_{i} \neq 0 \& r_{j} \neq 0\right),\left(k_{1}=0 \Leftrightarrow r_{1}=0\right), k_{i}, m_{i}, r_{i}, n_{i}, s \in\{0,1,2, \ldots\}\right\} .
\end{gathered}
$$

Запись вида $y^{0}$ означает отсутствие сомножителя $y^{0}$ в соответствующем произведении. Несложно заметить, что $\theta-$ конгруэнция на $F$. Отсюда $\theta_{A} \subseteq \theta$. Следовательно, $x / \theta_{A} \subseteq x / \theta$. Исследуем смежный класс $x / \theta$.

Возьмем произвольный элемент $y \in x / \theta, y \neq x$. Так как $(x, y) \in \theta$, элементы $x, y$ можно представить в следующем виде:

$$
x=x^{k_{1}} a^{m_{1}} b^{n_{1}} x^{k_{2}} a^{m_{2}} b^{n_{2}} \ldots x^{k_{s}} a^{m_{s}} b^{n_{s}}, \quad y=x^{r_{1}} a^{m_{1}} b^{n_{1}} x^{r_{2}} a^{m_{2}} b^{n_{2}} \ldots x^{r_{s}} a^{m_{s}} b^{n_{s}} .
$$

Длина элемента из $F$ - это сумма чисел вхождений $a$ и $b$ в запись этого элемента. В силу того, что существует $i$, для которого $k_{i} \neq 0$, длина элемента $x^{k_{1}} a^{m_{1}} b^{n_{1}} x^{k_{2}} a^{m_{2}} b^{n_{2}} \ldots x^{k_{s}} a^{m_{s}} b^{n_{s}}$ свободной полугруппы равна длине элемента $x$ тогда и только тогда, когда $k_{i}=1$ и все остальные показатели в этом произведении равны 0. Отсюда получаем, что $y=x^{r_{1}+\cdots+r_{s}}$, т. е. $y \in A$. Итак, доказали, что $x / \theta=A$, поэтому $x / \theta_{A}=A \in \mathscr{P}$. Значит, $F \in L_{1}(\mathscr{P})$.

В качестве $B$ возьмем любую простую некоммутативную 2-порожденную полугруппу, содержащую такой элемент $z$, что $z^{2} \neq z$ (например, знакопеременную группу $A_{5}$, рассматриваемую как полугруппу). Тогда $\theta_{\langle z\rangle} \neq \Delta$, откуда $z / \theta_{\langle z\rangle}=B \notin \mathscr{P}$, т. е. $B \notin L_{1}(\mathscr{P})$. Но полугруппа $B$ является гомоморфным образом свободной полугруппы $F$. Сказанное означает, что $L_{1}(\mathscr{P})$ не является многообразием. Теорема доказана.

\section{ЛИТЕРАТУРА}

1. Kappe L. C. On Levi-formations // Arch. Math. 1972. V. 23, N 1. P. 561-572.

2. Levi $F$. $W$. On groups in which the commutator operation satisfies certain algebraic conditions // J. Indian Math. Soc. 1942. V. 6. P. 87-97.

3. Morse R. F. Levi-properties generated by varieties // Contemp. Math. 1994. V. 169. P. 467-474.

4. Будкин А. И. Квазимногообразия Леви // Сиб. мат. журн. 1999. Т. 40, № 2. С. 266-270.

5. Kappe L. C., Morse R. F. Levi-properties in metabelian groups // Contemp. Math. 1990. V. 109. P. 59-72.

6. Kappe L. C., Morse R. F. Groups with 3-abelian normal closures // Arch. Math. 1988. V. 51, N 2. P. 104-110.

7. Будкин А. И. О классах Леви, порожденных нильпотентными группами // Алгебра и логика. 2000. Т. 39, № 6. С. 635-647.

8. Будкин А. И., Таранина Л. В. О квазимногообразиях Леви, порожденных нильпотентными группами // Сиб. мат. журн. 2000. Т. 41, № 2. С. 270-277.

9. Лодейщикова В. В. О квазимногообразиях Леви экспоненты $p^{s} / /$ Алгебра и логика. 2011. Т. 50, № 1. С. 26-41.

10. Лодейщикова В. В. О квазимногообразиях Леви, порожденных нильпотентными группами // Изв. АлтГУ. 2009. Т. 61, № 1. С. 26-29.

11. Лодейщикова В. В. Об одном классе Леви экспоненты $2 p / /$ Изв. АлтГУ. 2014. Т. 81, № $1 / 2$. C. $45-51$.

12. Лодейщикова В. В. Об одном квазимногообразии Леви экспоненты $8 / /$ Изв. АлтГУ. 2010. Т. 65 , № $1 / 2$. С. 42-45.

13. Лодейщикова В. В. О классах Леви, порожденных нильпотентными группами // Сиб. мат. журн. 2010. Т. 51, № 6. С. 1359-1366.

14. Лодейщикова В. В. Об одном многообразии Леви экспоненты $2 p / /$ Изв. АлтГУ. 2015. Т. 85 , № $1 / 1$. С. $84-88$. 
15. Лодейщикова В. В. О классе Леви, порожденном почти абелевым квазимногообразием нильпотентных групп // Изв. АлтГУ. 2016. Т. 89, № 1. С. 148-151.

16. Шахова С. А. Об аксиоматическом ранге квазимногообразия $M^{p^{2}} / /$ Изв. АлтГУ. 2015. Т. 85 , № $1 / 2$. С. $179-182$.

17. Смирнов Д. М. Многообразия алгебр. Новосибирск: Наука; Сибирская издательская фирма, 1992.

18. Мальцев А. И. Алгебраические системы. М.: Наука, 1970.

Поступила в редакиию 23 октлбря 2018 г.

После доработки 23 октября 2018 г.

Принята к публикации 19 декабря 2018 г.

Будкин Александр Иванович

Алтайский гос. университет,

ул. Ленина, 61, Барнаул 656064

budkin@math.asu.ru 